\title{
Hypernatraemic dehydration in infants with acute gastroenteritis at King Edward VIII Hospital, Durban, South Africa
}

T Hariram, MB ChB, DCH (SA), FC Paed (SA); K L Naidoo, MB ChB, DCH (SA), FC Paed; S Ramji, MB ChB

Department of Paediatrics and Child Health, University of KwaZulu-Natal, Durban, South Africa

Corresponding author: T Hariram (terishiapillay@gmail.com)

\begin{abstract}
Background. Acute gastroenteritis (AGE) is a leading cause of infant mortality, with hypernatraemic dehydration contributing to increased morbidity and mortality.

Objectives. To determine the prevalence of hypernatraemia secondary to AGE in admitted infants in Durban, South Africa. To describe the feeding choices, nutritional status and outcomes of these patients. To determine the association between admission sodium (Na) level, the rate of $\mathrm{Na}$ correction and clinical outcomes

Methods. A retrospective chart review was conducted on cases of hypernatraemic dehydration admitted in 2014 to a South African hospital. Serum Na levels were corroborated with National Health Laboratory Services results. Descriptive and analytical statistics were done using Statistical Package for Social Sciences version 22.

Results. A $12.3 \%$ prevalence of hypernatraemia $(n=41 / 334)$ was found. The majority of infants were formula-fed $(76 \%)$ with a $21 \%$ incidence of malnutrition and 66\% HIV exposure rate in this cohort. A high rate of neurological abnormalities (63\%), and a $4.9 \%$ case fatality rate was found. Shock on admission was present in $92 \%$ of patients who developed severe neurological complications. The mean admission Na was higher in those with severe neurological complications ( $164.2 \mathrm{v} .158 .4 \mathrm{mmol} / \mathrm{L}, p=0.08)$. The mean rate of Na change was not faster in those with severe neurological morbidity $(0.61 \mathrm{v} .0 .91 \mathrm{mmol} / \mathrm{L} / \mathrm{hr} ; p=0.1)$.

Conclusion. Hypernatraemic dehydration remains a significant problem in South Africa. High rates of formula feeding may be a contributory factor and the correlation with HIV infection needs investigation. Poor neurological outcomes were noted particularly in those patients presenting with hypernatraemia and shock. Although the mean admission Na level was higher in patients with severe neurological complications, this was not statistically significant in this sample. This study supports the hypotheses that neurological complications in diarrhoea-related hypernatraemia are largely associated with the severity of the dehydration that occurs prior to presentation rather than following rehydration.
\end{abstract}

S Afr J Child Health 2018;12(1):10-14. DOI:10.7196/SAJCH.2018.v12i1.1424

Approximately $76 \%$ of deaths in children under 5 years of age occur within the first 12 months of life, ${ }^{[1]}$ with diarrhoeal disease ranked as one of the leading causes of infant mortality in South Africa (SA) ${ }^{[1,2]}$ Hypernatraemic dehydration as a complication of diarrhoeal disease, contributes to the morbidity and mortality of affected patients, ${ }^{[3]}$ with a prevalence of $6.4 \%$ to $13.7 \%$, respectively, reported in developing

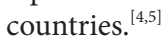

Diarrhoea-related hypernatraemic dehydration occurs as a result of a negative water balance, with water loss exceeding sodium $(\mathrm{Na})$ loss. ${ }^{[6]}$ Salt poisoning - commonly due to incorrect constitution of homemade oral rehydration therapy (ORT) and over-concentrated formula feeds - is also an important factor in the aetiology of hypernatraemia. ${ }^{[7]}$

Acute onset hypernatraemia increases serum osmolality, causing fluid to shift from the intracellular to the extracellular space, leading to cerebral cell dehydration and shrinkage. This can perpetuate structural changes resulting in intracranial haemorrhages. As a means of adaptation to gradual onset hypernatraemia, the brain increases its intracellular content of idiogenic osmoles, thus regaining its cell volume.$^{[3]}$ In this event, rapid correction of the $\mathrm{Na}$ with hypotonic intravenous fluids can lead to cerebral oedema.

Thus, neurological abnormalities are common, ranging from irritability and tone disturbances to seizures and coma. ${ }^{[3]}$ Mortality rates vary between $3 \%$ and $13 \%,{ }^{[8]}$ and hypernatraemic dehydration has been implicated as a predictor of death in children younger than 5 years old admitted with acute gastroenteritis (AGE). ${ }^{[9]}$
Despite high morbidity and mortality rates, the management of hypernatraemic dehydration is still not standardised in national guidelines. Institutions and teaching hospitals have developed individually tailored protocols developed by clinicians over many years. In KwaZulu-Natal (KZN), where AGE rates remain high, such a protocol has been in place for the past decade in Durban hospitals, including King Edward VIII Hospital (KEH), which is the major teaching hospital in the province. ${ }^{[10]}$ There are no published randomised controlled trials that clearly document the ideal choice of intravenous fluid, or the optimal rate of $\mathrm{Na}$ correction that can be undertaken without developing cerebral oedema, and there are conflicting results as to the correlation between the rate of fall of $\mathrm{Na}$ and clinical outcomes. ${ }^{[3,6]}$

To reduce its impact on the neurological morbidity of infants in SA, there is a need to further understand this condition and the factors that may contribute to such adverse outcomes. The aim of this study was to describe the extent of hypernatraemic dehydration in an HIV-endemic province in SA and to clarify if the major contributor to these outcomes is the severity of the hypernatraemia itself, or complications arising from rapid fluid correction.

The objectives of the study were: $(i)$ to determine the prevalence of hypernatraemia $(\mathrm{Na} \geq 150 \mathrm{mmol} / \mathrm{L})$ in all infants admitted with AGE at KEH from January until December 2014; (ii) to describe the feeding patterns, nutritional status and clinical outcomes of these infants; and (iii) to determine the association between admission $\mathrm{Na}$ level, rate of $\mathrm{Na}$ correction and clinical outcomes. 


\section{Methods}

This study was a retrospective chart review conducted at $\mathrm{KEH}$, a regional hospital within the Durban Metropolitan area in KZN. Purposive sampling was used to identify cases of hypernatraemic dehydration in infants younger than 12 months old. Utilising a physician-verified institution database, all cases of AGE in infants admitted between January and December 2014 were identified. The admission serum $\mathrm{Na}$ levels of these cases were traced via the National Health Laboratory Service (NHLS) Trakcare Web Results. Those with an admission $\mathrm{Na}$ level $\geq 150 \mathrm{mmol} / \mathrm{L}$ were included in the study. This two-step process aimed to prevent any omission of cases of hypernatraemia that might have not been documented in the admission database. The inpatient charts of identified cases were retrieved from the KEH medical records department for detailed clinical review. Demographic characteristics of each patient, nutritional status, feeding choice, as well as clinical data, including documented neurological symptoms, signs and outcomes (length of stay or mortality) were captured. Nutritional status was based on the weight-for-height $Z$-score, the presence of oedema and visible severe wasting. A $10 \%$ adjustment in admission weight was made to correct for the degree of dehydration. The serum $\mathrm{Na}$ on admission and subsequent Na levels during the course of inpatient management, together with their sampling times, was used to calculate the average rate of $\mathrm{Na}$ reduction until correction $(<150 \mathrm{mmol} / \mathrm{L})$ within the first 24 hours of admission.

To improve accuracy and minimise inter-observer variability, the clinical examination findings of the attending specialist paediatrician

\section{Results}

There were 1422 infants admitted to KEH in 2014, of whom 334 (23.5\%) had an admission diagnosis of AGE. The prevalence of hypernatraemic dehydration secondary to AGE in infants was found to be $12.3 \%$ ( $n=41 / 334$ cases). Three of the 41 patient files could not be traced, leaving 38 patients eligible for chart review. The sample population consisted of $84 \%$ infants under the age of 6 months $(n=32 / 38)$ and $16 \%$ between 6 to 12 months of age $(n=6 / 38) ; 55 \%$ were male $(n=21 / 38)$ and $45 \%$ female $(n=17 / 38)$. ORT had been used in $55 \%$ of patients $(n=21)$ prior to presentation, $8 \%$ of patients $(n=3)$ had not used ORT and $37 \%$ of the patient files $(n=14)$ did not document whether ORT had been used or not. An initial clinical assessment of shock was present in $45 \%$ of the patients $(n=17)$, of whom $65 \%$ also experienced a severe neurological complication. The HIV status of all infants with AGE is described in Table 1.

The hypernatraemic cohort was noted to have a $66 \%$ HIV exposure rate in comparison with $47 \%$ in those infants admitted for AGE without hypernatraemia, and this was a statistically significant difference $(p=0.03)$. Table 2 lists the nutritional status and documented feeding choices and practices of the sampled cohort.

With regards to nutrition and feeding, $21 \%$ of hypernatraemic patients were classified as either moderate or severe acute malnutrition. The nutritional classification of patients admitted for AGE without hypernatraemia was recorded as $64 \%$ normal, $17 \%$ underweight and $19 \%$ severely underweight for age.

Seventy-six percent of the hypernatraemic cohort were exclusively formula fed with only $16 \%$ exclusively breastfed. Of those receiving

Table 1. HIV status of infants with AGE admitted to KEH in 2014

\begin{tabular}{lll}
\hline HIV status & AGE with hypernatraemia $\left(\boldsymbol{N = 4 1 )}{ }^{*} \boldsymbol{n}(\%)\right.$ & AGE without hypernatraemia $(\boldsymbol{N}=\mathbf{2 9 3}), \boldsymbol{n}(\%)$ \\
\hline Unexposed & $14(34)$ & $151(52)$ \\
Exposed, HIV-uninfected & $20(49)$ & $91(31)$ \\
Exposed, HIV-unknown & $4(10)$ & $33(11)$ \\
Exposed, HIV-positive & $3(7)$ & $16(5)$ \\
Unknown & 0 & $2(1)$
\end{tabular}

${ }^{*}$ The HIV status of the three patients with missing files was obtained from the hospital admission database.

served as the primary source of data related to clinical symptoms and signs. For the purposes of this study, we recorded and classified the presence of irritability and/or abnormal muscle tone during the period of admission as a mild neurological abnormality. The occurrence of seizures and/or encephalopathy classified the patient as having a severe neurological abnormality.

It is plausible for a single patient to have more than one documented neurological abnormality during their admission; therefore, each patient was classified based on the most severe complication they developed, with encephalopathy considered most severe on the spectrum. This eliminated any duplication of results during the process of data analysis.

Data were captured on an Excel spreadsheet and subsequently analysed using the Statistical Package for Social Sciences version 22 (IBM Corp., USA). Descriptive statistics, including frequencies, percentages, means, medians and standard deviations (SD) were used to summarise results. Independent Samples $t$-test or the MannWhitney test was used to test for association between admission serum $\mathrm{Na}$ level, the rate of $\mathrm{Na}$ correction and outcomes. The level of significance was set at 0.05 .

\section{Ethics}

Ethical approval was obtained from the Biomedical Research Ethics Committee of the University of KwaZulu-Natal (ref. no. BE266/15).
Table 2. Nutritional status and feeding practices

\begin{tabular}{ll}
\hline & $\boldsymbol{n}(\%)$ \\
\hline Nutritional status $(N=38)$ & $30(79)$ \\
Normal nutrition & $3(8)$ \\
Moderate acute malnutrition & $5(13)$ \\
Severe acute malnutrition & \\
Feeding practices $(N=38)$ & $6(16)$ \\
Exclusive breastfeeding & $29(76)$ \\
Exclusive formula feeding & $3(8)$ \\
Mixed breast- and formula feeding & \\
Dilution of formula feeds $(N=32)$ & $3(9)$ \\
Correct & $3(9)$ \\
Incorrect & $26(82)$ \\
Not documented & \\
Reasons for not exclusively breastfeeding & $3(9)$ \\
Scholar & $5(16)$ \\
Returned to work & $10(31)$ \\
HIV-positive mother & $2(6)$ \\
Maternal or infant illness/medication & $5(16)$ \\
Insufficient breastmilk & $7(22)$ \\
Not documented &
\end{tabular}


formula feeds, $18 \%$ of patient caregivers were questioned about their formula dilution practices. Table 3 describes the clinical outcomes of the sampled cohort.

The majority (90\%) of patients were discharged from KEH and 2 patients $(4.9 \%)$ were transferred to an intensive care unit. The mortality rate of infants with hypernatraemic dehydration at KEH was $4.9 \%$. Both patients who demised had an initial clinical assessment of shock, as well as co-morbid conditions, one of which was severe sepsis with disseminated intravascular coagulation and the other was a chromosomal anomaly.

Neurological abnormalities were documented in $63 \%$ of reviewed infants, with $32 \%$ developing seizures and/or encephalopathy. Of those who developed seizures, $55 \%$ were preceded by irritability and/ or abnormal muscle tone. Of those wjo developed encephalopathy, $88 \%$ had preceding seizures. An initial clinical assessment of shock was present in $92 \%$ of patients who developed severe neurological complications. The association between admission serum $\mathrm{Na}$ level and neurological outcome is shown in Table 4.

The mean (SD) admission $\mathrm{Na}$ level for the sample population was $160.2(9.49) \mathrm{mmol} / \mathrm{L}$ (range 150 to 181). A higher admission serum $\mathrm{Na}$ level was seen in those who developed severe neurological abnormalities; however, this association was not statistically significant.

There was no statistically significant difference between the median admission $\mathrm{Na}$ of those who died $(n=2)$ and those who

\section{Table 3. Clinical outcomes}

\begin{tabular}{ll}
\hline Outcomes $(\boldsymbol{N}=\mathbf{4 1})^{\star}$ & $\boldsymbol{n}(\%)$ \\
\hline Discharged & \\
$\quad \leq 7$ days & $24(58.5)$ \\
$>7$ days & $13(31.7)$ \\
Transferred to PICU & $2(4.9)$ \\
Demised & $2(4.9)$ \\
Spectrum of neurological abnormalities $(N=38)$ & \\
Nil & $14(37)$ \\
Irritability and/or abnormal muscle tone only & $12(31.5)$ \\
Seizures & $4(10.5)$ \\
Seizures progressing to encephalopathy & $7(18)$ \\
Encephalopathy & $1(3)$
\end{tabular}

PICU $=$ paediatric intensive care unit.

*The outcomes of the three patients with missing files was obtained from the hospital admission database. survived ( $n=36)$ (160.5 v. $157.5 \mathrm{mmol} / \mathrm{L}, p=1$ using Mann-Whitney test).

The association between rate of $\mathrm{Na}$ correction and neurological outcome is shown in Table 5. The rate of Na correction ranged from -0.1 to $2.66 \mathrm{mmol} / \mathrm{L} / \mathrm{hr}$, with a mean (SD) of $0.81(0.53) \mathrm{mmol} / \mathrm{L} / \mathrm{hr}$ ( $n=37$, as one patient had only an admission serum Na record with no subsequent measurements).

Severe neurological complications were not associated with a faster mean rate of $\mathrm{Na}$ correction. The sample population was also grouped into three categories according to rate of $\mathrm{Na}$ correction: $<1 \mathrm{mmol} / \mathrm{L} / \mathrm{hr} ; 1-2 \mathrm{mmol} / \mathrm{L} / \mathrm{hr}$; and $>2 \mathrm{mmol} / \mathrm{L} / \mathrm{hr}$. The categories were compared with outcomes. This showed that $70 \%$ of all patients had a rate of $\mathrm{Na}$ correction $<1 \mathrm{mmol} / \mathrm{L} / \mathrm{hr}$. Of those with a rate of $\mathrm{Na}$ correction $\geq 1 \mathrm{mmol} / \mathrm{L} / \mathrm{hr}, 18 \%$ developed a severe neurological complication. There was no statistically significant difference in the development of neurological complications between the groups ( $p=0.489$ using Fisher's exact test). Both patients who demised had severe neurological morbidity, and both had a rate of $\mathrm{Na}$ correction $<1 \mathrm{mmol} / \mathrm{L} / \mathrm{hr}$.

\section{Discussion}

This study highlights the persistent burden of hypernatraemic dehydration secondary to AGE in SA. While the developed world now reports a decline in cases of hypernatraemic dehydration, ${ }^{[11]}$ the prevalence rate of $12.3 \%$ in our study remains similar to that of earlier studies conducted in developing countries. ${ }^{[4,5]}$

The incidence of hypernatraemia is said to increase with decreasing age, ${ }^{[4,5,10]}$ as was the case in this study, with $84 \%$ of infants being under the age of 6 months. The decreased thirst response, as well as the inability to access adequate amounts of free water despite ongoing losses, contributes to this particular age group being identified as high-risk. ${ }^{[3]}$

The hypernatraemic cohort in this study had a 66\% HIV exposure prevalence, compared with $47 \%$ in those infants admitted for AGE without hypernatraemia, and this was a statistically significant difference. Both these results were also noted to be higher than the provincial HIV exposure prevalence of $40.1 \%$ in $\mathrm{KZN} .{ }^{[12]}$ It has been shown that HIV exposed uninfected infants have an increased risk of infectious morbidity, particularly when born to mothers with advanced disease, ${ }^{[13,14]}$ and this could explain the increased prevalence among these infants with diarrhoeal disease. This study, however, describes a higher HIV exposure prevalence in relation to hypernatraemic dehydration, warranting further studies in this regard.

Table 4. Association between admission serum Na level (mmol/L) and severe neurological outcomes

\begin{tabular}{lllll}
\hline & & \multicolumn{3}{c}{ Admission serum Na (mmol/L) } \\
\cline { 3 - 5 } Clinical outcome $(\boldsymbol{N}=\mathbf{3 8})$ & $\boldsymbol{n}(\%)$ & Mean & SD & $\mathbf{9 5 \%} \mathbf{C I}$ \\
\hline No severe neurological abnormality noted & $26(68.4)$ & 158.4 & 7.9 & $155.2-161.6$ \\
Seizures and/or encephalopathy present & $12(31.6)$ & 164.2 & 11.6 & $156.8-171.6$ \\
$\mathrm{SD}=$ standard deviation; $\mathrm{CI}=$ confidence interval. & & & & \\
${ }^{*} p$-value (two sample $t$-test) $=0.08$. & & & &
\end{tabular}

Table 5. Association between rate of Na correction $(\mathrm{mmol} / \mathrm{L} / \mathrm{hr})$ and severe neurological outcomes

\begin{tabular}{|c|c|c|c|c|}
\hline \multirow[b]{2}{*}{ Clinical outcome $(N=37)$} & \multirow[b]{2}{*}{$n(\%)$} & \multicolumn{3}{|c|}{ Rate of $\mathrm{Na}$ correction $(\mathrm{mmol} / \mathrm{L} / \mathrm{hr})$} \\
\hline & & Mean & SD & $95 \% \mathrm{CI}$ \\
\hline No severe neurological abnormality noted & $25(67.6)$ & 0.91 & 0.58 & $0.67-1.14$ \\
\hline Seizures and/or encephalopathy present & $12(32.4)$ & 0.61 & 0.37 & $0.38-0.84$ \\
\hline
\end{tabular}


Due to $31 \%$ of mothers using formula feeds indicating that their HIV status was the reason for their feeding choice, and as formula feeds are a recognised risk factor for the development of hypernatraemic dehydration, ${ }^{[7,8,15]}$ we considered the effect of these feeding choices on HIV exposure prevalence and hypernatraemia; however, when we compared the HIV-exposed with the HIV-unexposed patients within the hypernatraemic cohort, we found an equally high number of patients who were exclusively formula-fed (76\% v. $77 \%)$.

The use of formula feeds in developing countries is increasing, with only $8.3 \%$ of SA infants under the age of 6 months reported to be exclusively breastfed. ${ }^{[16]} \mathrm{KEH}$ advocates the current national infant feeding strategy, which recommends exclusive breastfeeding for all infants until 6 months of age, including those born to HIV-positive mothers. In keeping with this, the distribution of free formula feeds to HIV-positive mothers was discontinued in $2011 .{ }^{[17]}$ Despite this, the low rate of exclusive breastfeeding was also apparent in our study at $\mathrm{KEH}$, with $76 \%$ of infants being formula-fed and $8 \%$ mixed-fed with both formula and breastmilk.

Although the majority of patients were formula-fed, we noted that $82 \%$ of caregivers were not questioned about formula dilution practices. Over-concentration of formula feeds leads to a high osmotic load resulting in hypernatraemia, ${ }^{[7,15]}$ so obtaining this information from caregivers is vital to identify such errors in feed dilution.

ORT serves as the first line management for the treatment of dehydration at the primary healthcare level; however, Dippenaar et al. ${ }^{[18]}$ showed that $14 \%$ of caregivers added too much salt to the homemade sugar-salt solution, which is a known risk factor for the development of hypernatraemia. We noted that $37 \%$ of caregivers were not asked about the use of ORT prior to admission. This should be reinforced as an important component of the history-taking process for any patient admitted with AGE, to identify errors in ORT constitution and prevent recurrences.

Approximately $21 \%$ of our study population was found to have moderate and severe acute malnutrition. This was an unexpected finding, as hypernatraemic dehydration is classically described in the well-nourished infant ${ }^{[19]}$ and its incidence is said to be inversely related to the degree of malnutrition. ${ }^{[4]}$ A study by Chisti et al. ${ }^{[20]}$ in Bangladesh reported that $28 \%$ of their hypernatraemic patients had severe wasting. This finding could be attributed to the higher rates of malnutrition in developing countries overall, as more than one-third of the infants admitted to KEH for AGE without hypernatraemia were also found to be underweight or severely underweight for age. Approximately two-thirds of patients in this study experienced abnormal neurological manifestations, of which $50 \%$ were seizures and/or encephalopathy. Robertson et al ${ }^{[8]}$ also reported a high incidence of seizures within their sample of hypernatraemic patients in Cape Town.

The mortality rate of $4.9 \%$ for hypernatraemic dehydration was similar to that of other studies. ${ }^{[8]}$ However, it was higher in comparison to the age-matched mortality rate of $1 \%$ for infants admitted to KEH for AGE without hypernatraemia. This highlights the increased risk of mortality in patients with hypernatraemic dehydration, but is it a higher admission $\mathrm{Na}$ level or a rapid rate of $\mathrm{Na}$ correction that contributes to this increased risk?

\section{Admission $\mathrm{Na}$ level and rate of $\mathrm{Na}$ correction}

The management of hypernatraemic dehydration is based on providing adequate free water to correct the serum $\mathrm{Na}$ level; $;{ }^{[3]}$ however, the rate at which it is corrected remains a point of contention. A study conducted in Cape Town found that rate of $\mathrm{Na}$ correction was not significantly associated with adverse outcomes ${ }^{[8]}$ In contrast, a Chinese study concluded that a faster rate of $\mathrm{Na}$ correction was a significant risk factor for the development of cerebral oedema. ${ }^{[21]}$

In the face of these conflicting study results, no definitively safe rate of correction has yet been advocated. Moritz and Ayus ${ }^{[3]}$ recommend that, unless symptoms of hypernatraemic encephalopathy are already present, a rate of correction not exceeding $1 \mathrm{mmol} / \mathrm{L} / \mathrm{hr}$ is reasonable. ${ }^{[19]}$

Another recommendation by Duggal et al.$^{[22]}$ is to factor in the rate of development of the hypernatraemia. A gradual onset requires slower correction at $0.5 \mathrm{mmol} / \mathrm{L} / \mathrm{hr}$, while an acute onset can tolerate faster correction, with a rate of $1 \mathrm{mmol} / \mathrm{L} / \mathrm{hr}$ deemed acceptable.

The experience at $\mathrm{KEH}$ has been to aim for a rate of $\mathrm{Na}$ correction between 0.5 and $1 \mathrm{mmol} / \mathrm{L} / \mathrm{hr}$ using a standardised hospital protocol that guides the fluid management of hypernatraemic dehydration. This protocol was developed by senior clinicians using their collective experience in the management of such cases over a number of years.

Standard ORT is used in cases of mild hypernatraemic dehydration. Where intravenous rehydration is required, fluids containing a $\mathrm{Na}$ concentration of approximately two-thirds that of the patient's $\mathrm{Na}$ level is used, at an initial rate of $10 \mathrm{~mL} / \mathrm{kg} / \mathrm{hr}$. This accounts for maintenance requirements, deficit and ongoing losses, and is continued until the level of hydration improves and the patient is able to tolerate adequate amounts of ORT. ${ }^{[10]}$

In this study, we found that $70 \%$ of hypernatraemic patients had a rate of $\mathrm{Na}$ correction $<1 \mathrm{mmol} / \mathrm{L} / \mathrm{hr}$, in keeping with recommendations in the literature.

Our study showed no statistically significant correlation between a faster rate of $\mathrm{Na}$ correction within the first 24 hours and adverse neurological outcomes and mortality. Of those with a rate of $\mathrm{Na}$ correction $\geq 1 \mathrm{mmol} / \mathrm{L} / \mathrm{hr}$, only $18 \%$ developed a severe neurological complication.

In the light of these findings, it appears that the hypernatraemia itself would be the more likely cause for adverse outcomes in these patients. This study showed that the mean admission $\mathrm{Na}$ level was higher in those with severe neurological morbidity and mortality; however, this association did not reach statistical significance.

We postulate that the development of neurological complications could be related to an interaction of factors prior to presentation, including the severity of the hypernatraemia together with the clinical presence of shock. In this study, 92\% of patients with severe neurological complications were in shock on presentation, highlighting it as an important factor in those with adverse outcomes. It is known that the degree of dehydration is often underestimated in hypernatraemic patients due to the relatively well-preserved intravascular volume. ${ }^{[6]}$ Thus, the signs of severe dehydration and shock occur late, and may initially be missed by caregivers. This leads to inadequate provision of ORT and delayed presentation, while intracellular cerebral dehydration prevails. Hypernatraemia and increasing plasma arginine vasopressin concentrations have also been shown to affect haemostatic function, thus promoting a hypercoaguable state, although the mechanism of this effect remains unclear. ${ }^{[23]}$ This association with an increased risk of thrombosis, together with the haemo-concentrated state induced by hypovolaemic shock, are risk factors for the development of cerebral sinus venous thrombosis, which can present with seizures and neurological abnormalities. ${ }^{[2]]}$ The use of neuroimaging would ideally differentiate between intracranial haemorrhages, thrombosis and cerebral oedema in patients who experience severe neurological complications during admission; however, this is not always feasible in resource-limited settings.

\section{Study limitations}

As this was a retrospective study, data were obtained from information documented in patient files, which were often poorly recorded with regard to ORT use and formula dilution.

There was a small sample size. There was a lack of definite timebased data for all neurological signs. There was no case control group in this study with which to make clear comparisons with the hypernatraemic group. 


\section{Conclusion and recommendations}

This study confirms that hypernatraemic dehydration related to AGE remains a significant challenge in South Africa, with a prevalence of $12.3 \%$ in a high HIV-exposed infant cohort. The predominant use of formula feeds, with low breastfeeding rates noted in these patients, may be a contributory factor. We also reaffirm the high rate of neurological morbidity and increased risk of mortality in hypernatraemic patients in this study. Mean admission $\mathrm{Na}$ was higher in cases with severe neurological complications and mortality, although this finding did not reach statistical significance. A faster rate of $\mathrm{Na}$ correction within the first 24 hours was not significantly associated with adverse outcome. This study suggests that the development of neurological complications could be related to an interaction of factors prior to presentation, including the severity of the hypernatraemia together with the clinical presence of shock. Larger multicentre trials are needed to further delineate these associations, to assist with management guidelines and reduce complications.

We recommend that greater emphasis should be placed on the pre-hospital management of patients with AGE, including educating caregivers on the correct use and constitution of ORT, appropriate practices and quick referral for help, to prevent the development of hypernatraemia and shock. Formula-fed infants should be identified as at risk for hypernatraemia. This study highlighted the poor documentation of ORT use and constitution, as well as formula dilution practices in patient records at the institution where the study took place. We suggest that existing admission templates be altered to remind staff to enquire about the relevant history in AGE cases.

In the management of infants with hypernatraemia following AGE, healthcare workers should aim for a reduction in serum $\mathrm{Na}$ between 0.5 and $1 \mathrm{mmol} / \mathrm{L} / \mathrm{hr}$, as per recommendations in the literature ${ }^{[3,22]}$ to prevent any added risk of cerebral oedema.

Acknowledgements. Catherine Connolly for her assistance with data analysis.

Author contributions. TH was responsible for study design, data collection, data analysis and drafting of the manuscript. KN and SR supervised the study and reviewed the manuscript.

Funding. None.

Conflicts of interest. None.

1. South African Medical Research Council (SAMRC). Under-5 Mortality Statistics in South Africa: Shedding Some Light on the Trends and Causes 1997-2007. Pretoria: SAMRC, 2012. http://www.mrc.ac.za/bod/MortalityStatisticsSA.pdf (accessed on 22 May 2017).

2. Statistics South Africa (SSA). Mortality and Causes of Death in South Africa, 2015: Findings from Death Notification. Pretoria: SSA, 2016. http://www. statssa.gov.za/publications/P03093/P030932015.pdf (accessed 22 May 2017).

3. Moritz ML, Ayus JC. Preventing neurological complications from dysnatremias in children. Pediatr Nephrol 2005;20(12):1687-1700. https://doi.org/10.1007/ s00467-005-1933-6

4. Samadi AR, Wahed MA, Islam MR, Ahmed SM. Consequences of hyponatraemia and hypernatraemia in children with acute diarrhoea in Bangladesh. Br Med J (Clin Res Ed) 1983;286(6366):671-673. https://doi org/10.1136/bmj.286.6366.671

5. Eke F, Nte A. A prospective clinical study of patients with hypernatraemic dehydration. Afr J Med Med Sci 1996;25(3):209-212.
6. El-Bayoumi MA, Abdelkader AM, El-Assmy MM, Alwakeel AA, El-Tahan HM. Normal saline is a safe initial rehydration fluid in children with diarrhea-related hypernatremia. Eur J Pediatr 2012;171(2):383-388. https://doi.org/10.1007/ s00431-011-1559-6

7. Abu-Ekteish F, Zahraa J. Hypernatraemic dehydration and acute gastroenteritis in children. Ann Trop Paediatr 2002;22(3):245-249. https://doi. org/10.1179/027249302125001624

8. Robertson G, Carrihill M, Hatherill M, Waggie Z, Reynolds L, Argent A. Relationship between fluid management, changes in serum sodium and outcome in hypernatraemia associated with gastroenteritis. J Paediatr Child Health 2007;43(4):291-296. https://doi.org/10.1111/j.1440-1754.2007.01061.x

9. Chisti MJ, Pietroni MA, Smith JH, Bardhan PK, Salam MA. Predictors of death in under-five children with diarrhoea admitted to a critical care ward in an urban hospital in Bangladesh. Acta Paediatr 2011;100(12):e275-279. https:// doi.org/10.1111/j.1651-2227.2011.02368.x

10. Naidoo KL, Ramji S. Acute gastroenteritis with hypernatraemic dehydration in children - a guide to management. S Afr Pediatric Rev 2007;4(2):19-21.

11. Moritz ML, Ayus JC. The changing pattern of hypernatremia in hospitalized children. Pediatrics 1999;104(3):435-439. https://doi.org/10.1213/ ane.0b013e3181f70826

12. National Department of Health (NDoH). The 2013 National Antenatal Sentinel HIV Prevalence Survey South Africa. Pretoria: NDoH, 2014. https:// www.health-e.org.za/wp-content/uploads/2016/03/Dept-Health-HIV-HighRes-7102015.pdf (accessed 22 May 2017).

13. Cooke ML, Nel ED, Cotton MF. Pre-hospital management and risk factors in children with acute diarrhoea admitted to a short-stay ward in an urban South African hospital with a high HIV burden. S Afr J Child Health 2013;7(3):84-87. https://doi.org/0.7196/SAJCH.472

14. Marinda E, Humphrey JH, Iliff PJ, et al. Child mortality according to maternal and infant HIV status in Zimbabwe. Pediatr Infect Dis J 2007;26(6):519-526. https://doi.org/10.1097/01.inf.0000264527.69954.4c

15. Taitz LS. Solute and calorie loading in young infants: Short- and longterm effects. Arch Dis Child 1978;53(9):697-700. https://doi.org/10.1136/ adc.53.9.697

16. Republic of South Africa Department of Health/Medical Research Council. South African Demographic Health Survey 2003. http://www.mrc.ac.za/bod/ sadhs.htm (accessed 22 May 2017)

17. Ijumba P, Doherty T, Jackson D, et al. Free formula milk in the prevention of mother-to-child transmission programme: Voices of a peri-urban community in South Africa on policy change. Health Policy Plan 2013;28(7):761-768. https://doi.org/10.1093/heapol/czs114

18. Dippenaar H, Joubert G, Nel R, Bantobetse ML, Opawole AA, Roshen KS. Homemade sugar-salt solution for oral rehydration: Knowledge of mothers and caregivers. SA Fam Pract 2005;47(2):51-53. https://doi.org /10.1080/20786204.2005.10873188

19. Diedericks RJ. Fluid therapy in the emergency unit. CME 2013;31(1):21-23.

20. Chisti MJ, Ahmed T, Ahmed S, et al. Hypernatraemia in children with diarrhea: Presenting features, management, outcome, and risk factors for death. Clin Pediatrics 2016;55(7):654-663. https://doi.org/10.1177/0009922815627346

21. Fang C, Mao J, Dai Y, et al. Fluid management of hypernatraemic dehydration to prevent cerebral oedema: A retrospective case control study of 97 children in China. J Paediatr Child Health 2010;46(6):301-303. https://doi.org/10.1111/ j.1440-1754.2010.01712.x

22. Duggal AK, Yadav P, Agarwal AK, Rewari BB. Clinical approach to altered serum sodium levels. JIACM 2006;7(2):91-103.

23. Grant PJ, Tate GM, Hughes JR, Davies JA, Prentice CR. Does hypernatraemia promote thrombosis? Thromb Res 1985;40(3):393-399.

24. Hashmi M, Wasay M. Caring for cerebral venous sinus thrombosis in children. J Emerg Trauma Shock 2011;4(3):389-394. https://doi.org/10.4103/09742700.83870

25. Chouchane S, Fehri H, Chouchane C, et al. Hypernatremic dehydration in children: Retrospective study of 105 cases. Arch Pediatr 2005;12(12):16971702 .

Accepted 7 August 2017. 